\title{
Sostenibilidad ambiental en la minería de materiales aluviales: el caso de Rio Negro, Dibulla, Colombia
}

\author{
Vilmer Y. Torres, Yessica M. Ramírez y Danny D. López \\ Facultad de Ingeniería, Universidad de La Guajira, Km. 5 salida a Maicao, Riohacha-Colombia. \\ (Correo-e: dlopezj@uniguajira.edu.co; vyeridtorres@uniguajira.edu.co; ymariaramirez@uniguajira.edu.co)
}

Recibido Mar. 22, 2021; Aceptado May. 24, 2021; Versión final Jun. 22, 2021, Publicado Dic. 2021

\begin{abstract}
Resumen
El presente estudio analiza la sostenibilidad ambiental en la minería de materiales aluviales en Rio Negro, Dibulla, Colombia. Se trata de un estudio no experimental, de diseño transeccional correlacional y de campo. Se empleó la técnica de observación mediante lista de chequeo. Se construyó un cuestionario y se utilizó la metodología de clasificación propuesta por Conesa para la valoración de impactos ambientales. Al aplicar la lista de chequeo, se obtuvo un total de 20 impactos ambientales que revelan la no sostenibilidad ambiental de la empresa minera. A partir de esta información se priorizaron los impactos y se proponen acciones ambientales para aquellos impactos negativos clasificados como moderados ( I $_{\mathrm{A}} \geq 25 \mathrm{y}<50$ ). Se concluye que la sostenibilidad ambiental en la empresa minera puede contribuir al mejoramiento de los procesos, convirtiéndose en una herramienta que le permite desarrollar sus actividades conforme a la normatividad.
\end{abstract}

Palabras clave: sostenibilidad ambiental; material aluvial; mediana minería; impacto; ambiental

\section{Environmental sustainability in the mining of alluvial materials: the case of Rio Negro, Dibulla, Colombia}

\begin{abstract}
This study assesses environmental sustainability for alluvial mining in Rio Negro, Dibulla, Colombia. This is a non-experimental field design study that is transactional and correlational. The observation technique is applied by means of a checklist and questionnaire construction. The Conesa classification methodology is employed to evaluate environmental impact. A total of 20 environmental impacts are obtained, revealing that mining is environmentally unsustainable. Based on this information, environmental impacts are prioritized and environmental actions are proposed for negative impacts classified as moderate ( $l_{\text {IA }} \geq 25$ and $\left.<50\right)$. It is concluded that environmental sustainability in mining can contribute to improving mining processes, thus becoming a tool for developing mining operations in accordance with regulations.
\end{abstract}

Keywords: environmental sustainability; alluvial material; medium mining; impact; valuation 


\section{INTRODUCCIÓN}

Durante las últimas décadas, las empresas han recibido innumerables críticas por su papel en los problemas ambientales (Wright y Nyberg, 2017), sin embargo, todavía existe una lista interminable de preocupaciones de insostenibilidad ambiental a las que estas organizaciones contribuyen. Muchas de las empresas se centran en la búsqueda de beneficios, sin tener en cuenta la responsabilidad de la sostenibilidad ambiental (Schaefer et al., 2020). La falta de unanimidad en los hallazgos de la investigación sobre el vínculo entre el desempeño económico y ambiental (Hang et al., 2019; Liu, 2020), ha creado un vacío en la literatura, haciendo que las organizaciones ignoren las serias preocupaciones creadas por la insostenibilidad ambiental (Wahab, 2021).

La sostenibilidad ambiental se define como la interacción responsable con el medio ambiente para evitar el agotamiento o la degradación de los recursos naturales y permitir la calidad ambiental a largo plazo (Sun et al., 2019). Este concepto de sostenibilidad ambiental se introdujo como un proceso costoso y se consideró como un freno para el desarrollo económico. Posteriormente, fue demostrada la problemática ambiental que generaba no introducir la sostenibilidad a los ámbitos económicos, sociales y culturales, fue allí donde se presentó la necesidad de futuras alternativas de desarrollo sostenible, que obligaban efectuar un uso racional de los recursos naturales renovables y no renovables, equilibrado, equitativo, conservacional y de protección (Gómez-Contreras, 2014). En este sentido, la humanidad se ha enfocado en la sostenibilidad ambiental, para aumentar las alternativas y generar nuevas estrategias que promuevan el desarrollo sostenible y ayuden a disminuir el agotamiento de los recursos naturales y al deterioro por causa de los procesos productivos.

Recientemente, ha habido un aumento en la conciencia pública sobre temas ambientales y los países han reconocido la necesidad de incrementar su desempeño ambiental (Cariola, 2020). Dentro de estas preocupaciones ambientales, un tema central se refiere a cómo se pueden administrar adecuadamente las empresas que operan en el sector minero. Dicho crecimiento viene, entre otras cosas, a través de la mejora de buenas prácticas, fomentando comportamientos rentables y aumentando el desempeño de las empresas que operan a pequeña y mediana escala en este sector (López et al., 2021). En este sentido, se hace necesario evaluar el desempeño ambiental en la pequeña y mediana minería. Además, de que las empresas mineras globales responsables, transfieran capacidades y tecnología a medida que el sector pyme evolucione (Mills, 2019).

A nivel mundial, la minería proporciona una fuente de sustento para millones de personas. Sin embargo, al mismo tiempo, el sector es predominantemente informal y está asociado con una variedad de desafíos sociales y ambientales persistentes, que incluyen pobreza extrema, deforestación, degradación de la tierra, contaminación ambiental y sedimentación de ríos (Hirons, 2020). Debido a estos impactos negativos, la industria minera ha estado bajo presión y ha comenzado a desarrollar estrategias para abordar las preocupaciones sobre los impactos sociales y ambientales de sus operaciones mineras (Ivic et al., 2021). Estas estrategias van dirigidas a promover en la dimensión ambiental, la utilización de los recursos de manera eficiente, limitar el consumo de recursos, reducir las emisiones de Gases de Efecto Invernadero (GEI), implementar recursos de energía renovable y reutilizar los residuos (Neto et al., 2018), y para la dimensión social, incluir factores sociales positivos como oportunidades de empleo, buenas condiciones laborales, oportunidades de aprendizaje e igualdad de género (Segerstedt y Abrahamsson, 2019).

En Colombia, el sector de pequeña y mediana minería de materiales aluviales se ha convertido en una forma de empleo rápida y factible, estas empresas solo necesitan mano obrera y una que otra maquinaria para explotar los minerales; sin embargo, esta minería, genera serios problemas ambientales al alterar las condiciones geométricas e hidráulicas de los ríos en el sitio de explotación y origina un desequilibrio entre los sedimentos transportados y la capacidad de transporte de la corriente. Estas alteraciones en las condiciones naturales del cauce generan a su vez procesos de erosión del lecho. La incisión del cauce puede inducir otros efectos, tales como, inestabilidad y erosión de las orillas, cambios en la morfología del cauce, variación en el tamaño de los materiales del lecho y ampliación del cauce (Bornachera et al., 2020).

En ese sentido, en Dibulla la minería de materiales aluviales se le añaden problemas ambientales relacionados con la manera antitécnica e irracional de explotar los recursos naturales, la falta de seguimiento por las autoridades ambientales, además de las condiciones geo-ambientales, sociales y políticas que hacen estos problemas más complejos. De este modo, la aplicación de la sostenibilidad ambiental puede ayudar a la reducción, control y/o mitigación de los impactos que trae consigo esta actividad y contribuir en el mejoramiento eficiente de este tipo de minería, de modo que, no se vea afectada la producción y la economía en estas mismas. La realización de este estudio radica en la importancia que tiene la minería de materiales aluviales, como actividad económica en el sector de la construcción en Dibulla y a nivel nacional. Por ello, es necesario involucrar estudios de impacto ambiental y aplicar la sostenibilidad ambiental en este tipo de minería, para mejorar, integrar y/o desarrollar prácticas que reduzcan el impacto ambiental negativo en sus operaciones. Además, de ser esto un requisito para poder tramitar las licencias ambientales (Resolución 1561 de 2019). 


\section{METODOLOGIA}

Se realizó un análisis descriptivo (Hernández et al., 2010), con un diseño no experimental, transeccional y de campo (Arias, 2012). La población corresponde a trabajadores de las diferentes áreas de una empresa dedicada a la extracción, procesamiento y comercialización de material aluvial (gravas y arenas) en Rio Negro, Dibulla, La Guajira, estudiándose todas las estrategias y alternativas que pudieran ser aplicadas a la sostenibilidad ambiental. Las muestras tomadas para el desarrollo del estudio representan el total de la población, brindando una alta confiabilidad. Las técnicas de recolección de información fueron de tipo observacional, además se aplicaron encuestas, teniendo en cuenta las características segmentarias y representativas. La población total se compone por 25 trabajadores de la empresa minera objeto de estudio, entre los cuales se destacan la gerencia, jefes de producción y recursos humanos, representante ambiental y operadores, de quienes se obtuvo la información que sirvió de base para el análisis y conclusiones del estudio.

En el presente estudio se construyó un cuestionario con 47 ítems, con un modelo de pregunta y respuesta abierta, que permitieron la búsqueda de información. Además, de una lista de chequeo que se emplea para identificar los de impactos ambientales (Molléri et al., 2020; Creswell, 2003). En el presente estudio la lista de chequeo permitió evaluar el impacto ambiental generado por las actividades que se llevan a cabo para explotar y procesar el material aluvial a los componentes ambientales. Así mismo, con la matriz por componente ambiental, propuesta por Fernandez-Vitora (1997), se determinaron las actividades susceptibles de producir impactos y se contó con la base de información primaria para realizar la jerarquización de impactos ambientales, mediante la utilización de una matriz de importancia utilizando el método de Conesa simplificado para ponderación de los impactos ambientales. Para la construcción de la matriz de importancia se tuvo en cuenta los valores obtenidos, para cada impacto. En la Tabla 1, se muestra las valoraciones del método de Conesa (Fernandez-Vitora, 1997), que determinan los valores cualitativos y cuantitativos, mostrando las caracterizaciones según la afectación y presencia del impacto.

Tabla 1: Valoración cualitativa y cuantitativa de impacto ambiental (Adaptada de Fernandez-Vitora, 1997).

\begin{tabular}{|c|c|c|c|c|c|}
\hline \multirow{3}{*}{ Naturaleza (NA) } & \multirow{2}{*}{ positivo } & \multirow{2}{*}{+} & \multirow{3}{*}{ Sinergia (SI) } & Sin sinergia & 1 \\
\hline & & & & Sinérgico & 2 \\
\hline & Negativo & - & & Muy sinérgico & 4 \\
\hline \multirow{4}{*}{ Intensidad (I) } & Bajo & 1 & \multirow{4}{*}{ Recuperabilidad (RE) } & Inmediato & 1 \\
\hline & Medio & 2 & & Media & 2 \\
\hline & Alto & 4 & & Mitigable & 4 \\
\hline & Muy alto & 8 & & Irrecuperable & 8 \\
\hline \multirow{4}{*}{ Extensión (EX) } & Puntual & 1 & \multirow{2}{*}{ Efecto (EF) } & Indirecto & 1 \\
\hline & Parcial & 2 & & Directo & 4 \\
\hline & Extensa & 4 & \multirow{2}{*}{ Acumulación (AC) } & Simple & 1 \\
\hline & Total & 8 & & Acumulativo & 4 \\
\hline \multirow{3}{*}{ Momento (MO) } & Largo & 1 & \multirow{3}{*}{ Periodicidad (PE) } & Irregular & 1 \\
\hline & Medio & 2 & & Periódico & 2 \\
\hline & Inmediato & 4 & & Continuo & 4 \\
\hline \multirow{3}{*}{ Persistencia (PR) } & Fugaz & 1 & \multirow{3}{*}{ Reversibilidad (RV) } & Corto & 1 \\
\hline & Temporal & 2 & & Mediano & 2 \\
\hline & Permanente & 4 & & Irreversible & 4 \\
\hline
\end{tabular}

Para la determinación de la importancia del impacto ambiental (lıA), se utilizó la (ecuación 1) de Conesa, para ponderar los impactos ambientales. La clasificación para cada impacto se determina mediante los siguientes valores: $<25$ impacto irrelevante; $\geq 25$ y $<50$ impacto moderado; $\geq 50$ y $<75$ impacto severo y $\geq 75$ impacto crítico.

$$
\mathrm{I}_{\mathrm{AA}}= \pm \sum(3 \mathrm{I}+2 \mathrm{EX}+\mathrm{MO}+\mathrm{PR}+\mathrm{RV}+\mathrm{RE}+\mathrm{SI}+\mathrm{AC}+\mathrm{EF}+\mathrm{PE})
$$

Para efectos de este estudio, se tuvo en cuenta la validez de contenido, realizado mediante la consulta técnica a expertos en las áreas de metodología de la investigación, sostenibilidad ambiental, evaluación de impacto ambiental y minería, buscando la validación del cuestionario, en relación con su contenido, efectividad, coherencia, entre otros aspectos. La confiabilidad no fue medida, por cuanto este estudio está sujeto a criterios propios como investigadores tanto en la observación en campo como para la selección de material documental.

\section{RESULTADOS Y DISCUSIÓN}

Actualmente, las empresas dedicadas a la explotación de materiales aluviales en Dibulla, Colombia se distribuyen en tres zonas principales: 1) zonas de mantenimiento (talleres), 2) zona de campamento (planta de procesamiento 
y centro de acopio) y 3) zona administrativa (oficinas, bodegas, área dispuesta para los trabajadores). Así mismo, el proceso que se lleva a cabo en la zona de campamento inicia con localización del punto de extracción y finaliza con la comercialización del material aluvial (Figura 1).

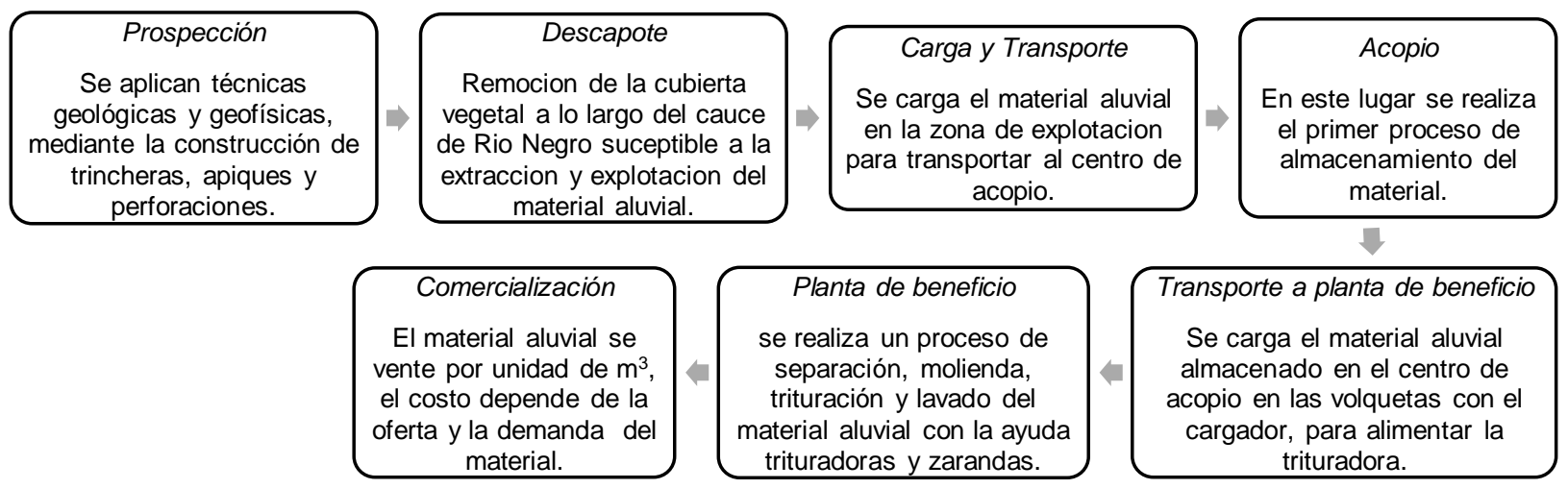

Fig. 1: Proceso de explotación en empresa que explota materiales aluviales en Dibulla, La Guajira

La empresa objeto de estudio tiene un volumen potencial de material aluvial de $7500 \mathrm{~m}^{3}$, con un área útil de explotación de $93.275 \mathrm{Ha}$ y un área promedio de explotación de $5000 \mathrm{~m}^{2}$ a una profundidad de $1.5 \mathrm{~m}$. El sistema de explotación que desarrolla la empresa es a cielo abierto, mediante el método por dársenas perpendiculares al cauce del rio, el cual consiste en la explotación de material aluvial que ha sido transportado y depositado por el rio, el cual se extraen utilizando una maquina Caterpillar $336 \mathrm{~d}$ y luego es transportado en volquetas que tienen una capacidad de $14 \mathrm{a} 15 \mathrm{~m}^{3}$ al centro de acopio.

Por otro lado, al aplicar la lista de chequeo se identificaron los impactos ambientales que afectan al componente ambiental y que son generados por las actividades llevadas a cabo para la explotación del material aluvial. En total se identificaron 20 impactos ambientales evidenciando que la actividad de descapote genera la mayoría de estos impactos ambientales. En la Tabla 2 se muestra una síntesis de la lista de chequeo, donde el proceso de identificación de impactos ambientales se aplicó a una empresa minera que explota material aluvial en Rio Negro, Dibulla, La Guajira.

Tabla 2: Lista de chequeo en empresa que explota materiales aluviales en Dibulla, La Guajira

\begin{tabular}{|c|c|c|c|c|c|c|c|}
\hline & \multicolumn{4}{|c|}{ Actividades } & \multirow{5}{*}{$\begin{array}{l}\stackrel{\mathscr{C}}{\frac{\mathbb{D}}{O}} \\
\frac{1}{2}\end{array}$} & \\
\hline & & Descapote & Transporte & Almacenamiento & Triturado & & \\
\hline \multirow{20}{*}{$\begin{array}{l}\overline{\bar{z}} \\
\overline{0} \\
\overline{0} \\
\overline{0} \\
\end{array}$} & Alteración del revestimiento vegetal & $\mathrm{X}$ & $\mathrm{X}$ & & & & \\
\hline & Pérdida de suelo fértiles & $\bar{X}$ & $\mathrm{X}$ & $\mathrm{X}$ & & & \\
\hline & Erosión & $\mathrm{X}$ & & & & & \\
\hline & Fuentes de contaminación & $\mathrm{X}$ & & & & \multirow{3}{*}{ 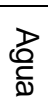 } & \multirow{17}{*}{ 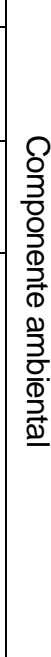 } \\
\hline & Estrés hídrico & $\mathrm{X}$ & $\mathrm{X}$ & & & & \\
\hline & Captaciones (caudal ecológico) & $\mathrm{X}$ & & & & & \\
\hline & Material particulado & & $\mathrm{X}$ & $\mathrm{X}$ & $\mathrm{X}$ & \multirow{3}{*}{$\stackrel{D}{\stackrel{D}{D}}$} & \\
\hline & Emisiones de gases tóxicos y no tóxicos & & $\mathrm{X}$ & & $\mathrm{X}$ & & \\
\hline & Dispersión del ruido & $\mathrm{X}$ & $\mathrm{X}$ & & $\mathrm{X}$ & & \\
\hline & Deforestación & $\mathrm{X}$ & $\mathrm{X}$ & & $\bar{x}$ & \multirow{11}{*}{ 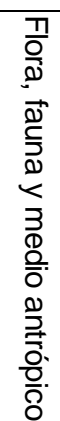 } & \\
\hline & Pérdida de biodiversidad & $\mathrm{X}$ & & & & & \\
\hline & Efectos en las especies endémicas & $\mathrm{X}$ & $\mathrm{X}$ & & $\mathrm{X}$ & & \\
\hline & Poca disponibilidad de agua & $\mathrm{X}$ & & & & & \\
\hline & Destrucción de hábitat & $\mathrm{X}$ & $\mathrm{X}$ & & $\mathrm{X}$ & & \\
\hline & Alteración de la cadena trófica & $\mathrm{X}$ & & & $\mathrm{X}$ & & \\
\hline & Uso inadecuado del territorio & $\mathrm{X}$ & & & $X$ & & \\
\hline & Inestabilidad en el terreno & $\mathrm{X}$ & $\mathrm{X}$ & & & & \\
\hline & Canales de drenaje & $\mathrm{X}$ & & & & & \\
\hline & Alteración del paisaje & $\bar{X}$ & & $\mathrm{X}$ & $\mathrm{X}$ & & \\
\hline & Destrucción de los sistemas naturales & $\mathrm{X}$ & & & $\mathrm{X}$ & & \\
\hline
\end{tabular}

Con ayuda del cuestionario se logró identificar que en las zonas de mantenimiento y campamento existe una alteración geomorfológica del cauce del rio, causado por la extracción de material aluvial y el tránsito vehicular en la zona de extracción. Haghnazar et al. (2020) indica que, dependiendo de la morfología del río y las características hidráulicas, su capacidad de transporte de sedimentos y el método de operación minera, la extracción de materiales del lecho del río puede afectar su ecosistema a través de la erosión del lecho del rio. Por otro lado, en la zona administrativa se evidencia la generación de residuos peligros y no peligrosos, por lo que se hace necesario el establecimiento de puntos de reciclajes o ecológicos que ayuden a hacer una buena separación 
y disposición de estos para contribuir con la sostenibilidad ambiental en la empresa. A continuación, se hace descripción de los indicadores que resaltan los impactos negativos generados al recurso suelo, aire, agua y biodiversidad en la empresa objeto de estudio, producto de las actividades y proceso llevado a cabo para explotar el material aluvial.

En cuanto al recurso suelo, se tiene que este es el factor más afectado en el proceso de explotación, seguido del recurso hídrico, es claro afirmar que las principales problemáticas ambientales son: la disminución del caudal, los cambios de la dinámica natural del sistema y alteración de la estructura del suelo por la extracción del material aluvial, ocasionados por el arranque del material que genera alteración de la geomorfología y pérdida física de suelo, estos problemas coinciden con los proporcionados por Dépret et al. (2008), en cuanto a los cambio físicos y geomorfológicos del canal de los ríos. También, en la empresa existen procesos erosivos, como la erosión eólica que impacta significativamente al suelo, debido a que las condiciones naturales actúan de manera abruptas en las zonas con poca presencia de vegetación, producto del descapote o remoción de cobertura vegetal que se realiza en la etapa de exploración.

En cuanto a la biodiversidad, la empresa da cumplimiento al programa de manejo de fauna y flora, donde se tiene prohibido la captura y caza de especies faunística, al igual que la tala de especies vegetativas. El representante ambiental de la empresa, indica que cuando se requiere talar, en consecuencia, de las actividades propias del proyecto, se expide el respectivo permiso de aprovechamiento forestal, ante la Corporación Autónoma de La Guajira (Corpoguajira). Así mismo, las instalaciones cuentan con una barrera viva perimetral, poseen un vivero de especies endémicas de la zona, realizan siembras de árboles en las áreas destinadas para rehabilitación y conservación en la zona minera, estas áreas se encuentran principalmente en la margen del rio. Una de sus actividades de recuperación es la reforestación de las áreas afectadas por su actividad o por las condiciones naturales del entorno.

Por su parte, la fauna sufre una afectación debido a los procesos mineros, en la visita a la empresa se pudieron detectar que factores como las horas de operación y el ruido producido por toda la maquinaria pesada, inciden en la migración de la fauna. Es preciso señalar que no existen estudios sobre la ictiofauna e hidrófitas, ni documento por parte de Corpoguajira donde se indique el tipo de flora y fauna acuática en presentes en el rio donde se realiza la explotación. Sin embargo, este tipo de minería presentan mayores valores de dominancia por especies acuáticas tolerantes a condiciones de turbidez. Córdoba et al. (2016), en su estudio reporta que ambientes creados por actividades mineras (ej. formación de ambientes lénticos temporarios debido a excavaciones) son utilizados como hábitats temporales por diversas especies de peces como Cyphocharax magdalenae, Roeboides dayi, Andinoacara latifrons y Poecilia caucana.

En cuanto al recurso hídrico, la empresa cuenta con un medidor de consumo de agua que permite controlar el uso del recurso hídrico, así mismo la fuente de abastecimiento corresponde a un pozo artesano, donde el agua se aprovecha para riego de vías, uso doméstico y para las necesidades de saneamiento básico. Sin embargo, la empresa no cuenta con un sistema de tratamiento de aguas residuales, en efecto cuenta con depósitos de aguas residuales (fosas sépticas cerradas) donde se efectúan los vertimientos de las aguas residuales generadas en la zona administrativa de la empresa. Por otra parte, se observó que en la zona de campamento existen transformaciones en la morfometría del cauce principal del rio, producto de excavaciones en la zona de explotación, lo que causa la destrucción del habitad, inmigración de especies acuáticas y contaminación del recurso hídrico (Haghnazar et al., 2020).

En cuanto a la contaminación atmosférica, se encontraron diversas problemáticas asociadas a la emisión de material particulado y $\mathrm{GEI}$, a pesar de las estrategias que se implementan para reducir la contaminación atmosférica, se evidencia las emisiones de GEI generadas a la atmósfera producto de los (fuentes móviles) y el transporte y triturado del material aluvial, generando a su vez cambios físicos cómo la poca visibilidad a causa de las nubes de polvo y por ende el cambio de entorno natural; por otra parte, químicamente se producen cambios en el estado natural del aire debido a que pierde pureza por la sinergia de las partículas de polvo con otros materiales como el dióxido de carbono, monóxido de carbono e hidrocarburos (Sawlani et al., 2020).

En consecuencia, este tipo de minería debe controlar todas aquellas alteraciones que puedan afectar directamente al ambiente, específicamente al aire, por ello, deben trabajar para disminuir las concentraciones de material particulado, la Resolución 2254 de 2017 estipula que los nivelas máximos permisibles para PM10 son de $50 \mu \mathrm{g} / \mathrm{m} 3$ y la empresa no está cumpliendo con ello. Actualmente, el material particulado emitido por la empresa es de $75 \mu \mathrm{g} / \mathrm{m}^{3}$, por lo que está sobrepasando los niveles máximos permisibles. De acuerdo, con el factor ruido ambiental la empresa objeto de estudio cuenta para sus operaciones de minería con un parque automotriz de 3 retroexcavadoras, 2 trituradoras y 27 volquetas de carga, las cuales operan durante 12 horas y son la fuente principal de generación de ruido ambiental, a causa del movimiento de los vehículos, uso de pitos y bocinas y también ruidos asociados a la zona de mantenimiento donde se usan taladros para desmontar las llantas de las máquinas. 
Por otro lado, en la Tabla 3 se muestra la matriz resultante del método de Conesa de la empresa objeto de estudio, donde se evaluaron los impactos ambientales generados a los componentes ambientales, producto de las actividades mineras, tales como: deterioro de la calidad del aire por la emisión de material particulado, ruido ambiental generado por maquinaria, alteración de la estructura del suelo por la extracción del material aluvial, deterioro de la calidad del agua por la presencia de materiales contaminantes en las maquinarias, alteración del cauce por la utilización de maquinaria, desplazamiento de especies animales por la generación de ruido, pérdida de especies vegetales por la movilización de maquinarias, afectación de la salud de las comunidades aledañas y trabajadores de la empresa, por la contaminación del aire y la generación de empleo. Estos problemas ambientales coinciden con los estudios realizados por Padmalal y Maya (2014) y Zhai et al. (2020), quienes indican que la extracción indiscriminada de arena y grava de los ríos impone daños irreparables a los ecosistemas fluviales, incluidas sus áreas de llanuras aluviales y de ríos. Por lo tanto, es una premisa esencial para incorporar la sostenibilidad ambiental y garantizar que el entorno ecológico circundante no se vea afectado por la extracción material aluvial.

Tabla 3: Matriz resultante del método de Conesa en empresa que explota materiales aluviales en Dibulla, La Guajira

\begin{tabular}{|c|c|c|c|c|c|c|c|c|c|c|c|c|c|c|}
\hline \multicolumn{2}{|c|}{ Factor ambiental } & \multirow[b]{2}{*}{ Impactos generados } & \multicolumn{12}{|c|}{ Valoración } \\
\hline Medio & Componente & & 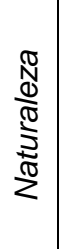 & 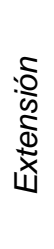 & $\begin{array}{l}\frac{\pi}{d} \\
\frac{d}{d} \\
\frac{D}{D} \\
\frac{D}{D} \\
0\end{array}$ & $\frac{\sqrt{2}}{\frac{\pi}{2}}$ & $\begin{array}{l}0 \\
\frac{0}{4} \\
\frac{d}{4}\end{array}$ & 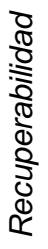 & 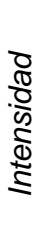 & 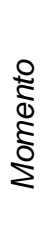 & 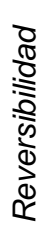 & 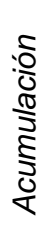 & $\begin{array}{l}\frac{\pi}{0} \\
\frac{\pi}{0} \\
\frac{0}{0} \\
0\end{array}$ & $I_{I A}$ \\
\hline \multirow{5}{*}{ Abiótico } & \multirow{2}{*}{ Aire } & $\begin{array}{l}\text { Deterioro de la calidad del aire por la } \\
\text { emisión de material particulado. }\end{array}$ & $(-)$ & 4 & 2 & 2 & 4 & 4 & 4 & 4 & 2 & 1 & 4 & 43 \\
\hline & & $\begin{array}{l}\text { Ruido ambiental generado por } \\
\text { maquinaria. }\end{array}$ & $(-)$ & 2 & 2 & 1 & 1 & 4 & 2 & 4 & 2 & 1 & 2 & 23 \\
\hline & Suelo & $\begin{array}{l}\text { Alteración de la estructura del suelo por } \\
\text { la extracción del material aluvial. }\end{array}$ & $(-)$ & 2 & 2 & 1 & 4 & 8 & 4 & 2 & 4 & 1 & 2 & 36 \\
\hline & \multirow[t]{2}{*}{ Agua } & $\begin{array}{l}\text { Deterioro de la calidad del agua por la } \\
\text { presencia de materiales contaminantes } \\
\text { en las maquinarias. }\end{array}$ & $(-)$ & 2 & 2 & 2 & 1 & 4 & 2 & 2 & 2 & 1 & 2 & 22 \\
\hline & & $\begin{array}{l}\text { Alteración del cauce por la utilización } \\
\text { de maquinaria. }\end{array}$ & $(-)$ & 2 & 2 & 1 & 4 & 4 & 2 & 2 & 2 & 1 & 2 & 24 \\
\hline \multirow{2}{*}{ Biótico } & Fauna & $\begin{array}{l}\text { Desplazamiento de especies animales } \\
\text { por la generación de ruido. }\end{array}$ & $(-)$ & 1 & 2 & 1 & 1 & 4 & 2 & 2 & 2 & 1 & 4 & 23 \\
\hline & Flora & $\begin{array}{l}\text { Pérdida de especies vegetales por la } \\
\text { movilización de maquinarias. }\end{array}$ & $(-)$ & 1 & 2 & 2 & 4 & 2 & 2 & 2 & 2 & 1 & 2 & 23 \\
\hline \multirow[t]{2}{*}{ Antrópico } & Social & $\begin{array}{l}\text { Afectación de la salud de las } \\
\text { comunidades aledañas y trabajadores } \\
\text { de la empresa, por la contaminación } \\
\text { del aire. }\end{array}$ & $(-)$ & 2 & 2 & 2 & 4 & 4 & 4 & 1 & 4 & 4 & 2 & 35 \\
\hline & Económico & Generación de empleo & $(+)$ & 4 & 2 & 1 & 4 & 1 & 8 & 2 & 2 & 1 & 2 & 39 \\
\hline
\end{tabular}

Al aplicar la Ecuación 1, se encontró que el nivel de importancia de los impactos negativos generados corresponde a: 1) deterioro de la calidad del aire por la emisión de material particulado: Moderado. 2) Ruido ambiental generado por maquinaria: irrelevante. 3) Alteración de la estructura del suelo por la extracción del material aluvial: Moderado. 4) Deterioro de la calidad del agua por la presencia de materiales contaminantes en las maquinarias: irrelevante. 5) Alteración del cauce por la utilización de maquinaria: irrelevante. 6) Desplazamiento de especies animales por la generación de ruido: irrelevante. 7) Pérdida de especies vegetales por la movilización de maquinarias: irrelevante. 8) Afectación de la salud de las comunidades aledañas y trabajadores de la empresa, por la contaminación del aire: Moderado. Así mismo, los impactos positivos corresponden a: 1) generación de empleo: Moderado. Teniendo en cuenta la valorización de los impactos ambientales identificados, se proponen acciones ambientales para aquellos impactos negativos clasificados como moderados ( $l_{1 A} \geq 25 \mathrm{y}<50$ ), cuyas acciones están encaminadas a promover la sostenibilidad ambiental en la empresa objeto de estudio (Tabla 4-6).

\section{CONCLUSIONES}

A partir de los resultados obtenidos, se pueden extraer las siguientes conclusiones: 1) con el desarrollo del presente estudio se pudo obtener información veraz acerca del estado y desarrollo actual de una empresa minera dedicada a la explotación de materiales aluviales en Rio Negro, Dibulla, Colombia, a partir de la utilización y seguimiento de un plan metodológico apoyado en la aplicación de cuestionario, listas de chequeo 
y jerarquización de impactos ambientales con el método de Conesa, 2) el estudio muestra que la explotación de materiales aluviales llevada a cabo por la empresa, presenta problemas ambientales asociados con la degradación del suelo, emisión de contaminantes atmosféricos, contaminación del recurso hídrico y evidente afectación a la biodiversidad; 3) la sostenibilidad ambiental en la minería de materiales aluviales permite la búsqueda de alternativas sostenibles para mantenerse dentro de los márgenes normativos y promover la gestión ambiental dentro de su área de influencia; 4) al determinar el nivel de importancia de los impactos ambientales, se concluye que en la empresa minera estudiada se requiere un nivel de importancia moderado, por consiguiente, la recuperación de los componentes ambientales, no requiere de prácticas correctoras intensivas.

Tabla 4: Control de emisiones de material particulado

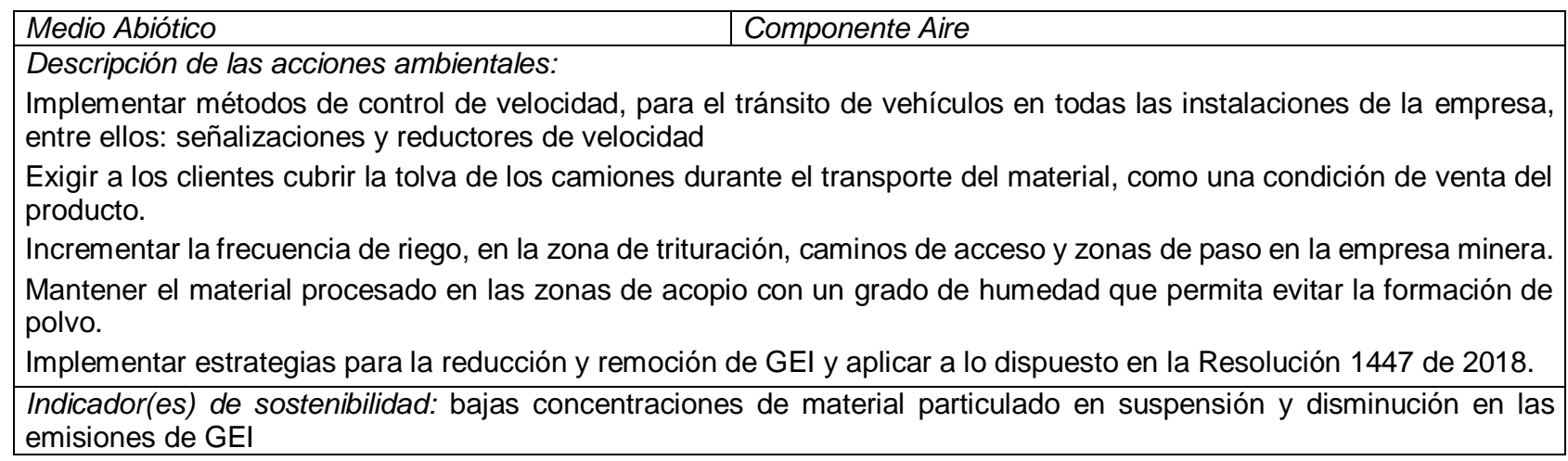

Tabla 5: Rehabilitación de la estructura del suelo causada por la extracción del material aluvial

\begin{tabular}{l}
\hline Medio Abiótico \\
\hline Descripción de las acciones ambientales: \\
Evitar excavaciones y remociones de suelo innecesarias. \\
El material aluvial removido que la empresa minera considera no útil para ser transformado debe ser dispuesto en el \\
área de extracción para estimular auto regeneración del suelo y reestablecer su estructura natural. También puede ser \\
utilizado para obras civiles dentro de la empresa. \\
Se deben establecer pilas con formas apropiadas y ajustadas al espacio disponible, teniendo presente no acumular \\
demasiado suelo en un mismo sitio. \\
\hline Indicador(es) de sostenibilidad: extracción de materia prima en excelentes condiciones y presencia de material aluvial \\
en zonas del rio ya explotadas.
\end{tabular}

Tabla 6: Disminución de riesgos laborales

\begin{tabular}{|l|l|}
\hline Medio Antrópico & Componente Social \\
\hline Descripción de las acciones ambientales: & \\
Verificar que los vehículos de transporte del material cuenten con el seguro obligatorio de accidentes de tránsito (SOAT). \\
Instalar señalizaciones de advertencia, seguridad y paso peatonal dentro de las instalaciones de la empresa. \\
Delegar a una persona que realice jornadas de monitoreo para verificar el uso adecuado de los elementos de protección \\
personal. \\
Desarrollar capacitaciones al personal en general sobre los factores de riesgos a los que están expuestos por el trabajo \\
en el que se desempeñan abarcando temáticas como salud ocupacional, seguridad industrial y calidad ambiental. \\
\hline Indicador(es) de sostenibilidad: reducción de accidentes laborales por jornadas de trabajo. \\
\hline
\end{tabular}

\section{REFERENCIAS}

Arias, F., El proyecto de Investigación, Introducción a la Metodología Científica, Editorial Episteme, Venezuela (2012)

Bornachera, K.P., Juvinao, D.D.L., y Jaramillo, A.H., Transferencia Tecnológica para la Producción Limpia en la Minería de Materiales Aluviales en La Guajira, Colombia, https://doi.org/10.17081/invinno.8.1.3535, Investigación e Innovación en Ingenierías, 8(1), 6-20 (2020)

Cariola, A., Fasano, F., La Rocca, M., y Skatova, E., Environmental Sustainability Policies and the value of debt in EU SMEs: Empirical evidence from the Energy Sector, https://doi.org/10.1016/j.jclepro.2020.123133, Journal of Cleaner Production, 1-12 (2020)

Córdoba, D., Vásquez, D., y otros 3 autores, Diversidad de peces en Sistemas Lóticos y Lentícos asociada al Bioma de Bosque Seco de Victoria, Caldas, Revista de Ciencias, ISSN: 2248-4000, 20(2), 61-78 (2016)

Creswell J.W., Research Design: Qualitative, Quantitative, and Mixed Methods Approaches, 3rd ed, SAGE Publiations Ltd., Londres, Nueva Delhi (2003) 
Dépret, T., Virmoux, C., y otros 10 autores, Lowland Gravel-Bed River Recovery through former Mining Reaches, the key role of sand, https://doi.org/10.1016/j.geomorph.2020.107493, Geomorphology, 373, 1-18 (2021)

Fernandez-Vitora, V., Guía metodológica para la evaluación del impacto ambiental, 3a ed., 1-412, Madrid, España (1997)

Gómez-Contreras, J.L., Del Desarrollo Sostenible a la Sostenibilidad Medioambiental, Revista Facultad de Ciencias Económicas: Investigación y Reflexión, ISSN: 0121-6805, 22(1), 115-136 (2014)

Haghnazar, H., Sangsefidi, Y., Mehraein, M., y Tavakol- Davani, H., Evaluation of Infilling and replenishment of River Sand Mining Pits, https://doi.org/10.1007/s12665-020-09106-z, Environ Earth Sci, 79(362), 1-18 (2020)

Hang, M., Geyer-Klingeberg, J., y Rathgeber, A.W., It is Merely a Matter of Time: A Meta-Analysis of the Causality between Environmental Performance and Financial Performance, https://doi.org/10.1002/bse.2215, Business Strategy and the Environment, 1-17 (2018)

Hernández, R., Fernández, C., y Baptista, P., Metodología de la Investigación, Mc Graw Hill, México (2010)

Hirons, M., How the Sustainable Development Goals Risk Undermining Efforts to address Environmental and Social issues in the Small-Scale Mining Sector, https://doi.org/10.1016/j.envsci.2020.08.022, Environmental Science \& Policy, $114,321-328(2020)$

Ivic, A., Saviolidis, N.M., y Johannsdottir, L., Drivers of sustainability practices and contributions to sustainable development evident in sustainability reports of European mining companies, https://doi.org/10.1007/s43621-021-00025y, Discover Sustainability 2(17), 1-20 (2021).

Liu, Z., Unraveling the Complex Relationship between Environmental and Financial Performance - A Multilevel Longitudinal Analysis, https://doi.org/10.1016/j.ijpe.2019.07.005, International Journal of Production Economics, 219, 328-340 (2020)

López, D.D., Melo, G.M., y Mendoza, D.L., Gestión logística en la industria salinera del departamento de La Guajira, Colombia, https://dx.doi.org/10.4067/S0718-07642021000100039, Información tecnológica, 32(1), 39-46 (2021)

Mills, T., Is Afghanistan's mineral wealth best left in the ground?, https://doi.org/10.1016/j.exis.2019.10.001, The Extractive Industries and Society, 7(1), 73-78 (2019)

Molléri, J.S., Petersen, K., y Mendes, E., An Empirically Evaluated Checklist for Surveys in Software Engineering https://doi.org/10.1016/j.infsof.2019.106240, Information and Software Technology, 119 (2020)

Neto, O.G.C., Pinto, L.F.R., Amorim, M.P.C., Giannetti, B.F., Almeida, C.M.V.B., A framework of actions for strong sustainability, https://doi.org/10.1016/j.jclepro.2018.06.067, Journal of Cleaner Production, 196, 1629-1643 (2018)

Padmalal, D., y Maya, K., Sand Mining: The World Scenario, https://doi.org/10.1007/978-94-017-9144-1_5, Environmental Science and Engineering, 57-80 (2014)

Resolución 1447, Reglamento del sistema de monitoreo, reporte y verificación de las acciones de mitigación, 62-72, Bogotá D.C., Colombia (2018)

Resolución 1561, Términos de referencia para la elaboración del Estudio de Impacto Ambiental (EIA), 1-28, Bogotá D.C., Colombia (2019)

Resolución 2254, Por la cual se adopta la norma de calidad del aire ambiente y se dictan otras disposiciones, 1-11, Bogotá D.C., Colombia (2017)

Sawlani, R., Agnihotri, R., y Sharma, C., Chemical and Isotopic Characteristics of PM2.5 over New Delhi from September 2014 to May 2015: Evidences for Synergy Between Air-Pollution and Meteorological Changes, https://doi.org/10.1016/j.scitotenv.2020.142966, Science of The Total Environment, 763, 1-16 (2020)

Schaefer, K., Corner, P.D., y Kearins, K., Social, Environmental and Sustainable Entrepreneurship Research, https://doi.org/10.1177/1086026615621111, Organization \& Environment, 28(4), 394-413 (2015)

Segerstedt, E., Abrahamsson, L., Diversity of livelihoods and social sustainability in established mining communities, https://doi.org/10.1016/j.exis.2019.03.008, The Extractive Industries and Society, 6(2), 610-619 (2019)

Sun, H., Mohsin, M., Alharthi, M., y Abbas, Q., Measuring Environmental Sustainability Performance of South Asia, https://doi.org/10.1016/j.jclepro.2019.119519, Journal of Cleaner Production, 251, 1-13 (2019)

Wahab, M., Is an Unsustainability Environmentally unethical? Ethics Orientation, Environmental Sustainability Engagement and Performance, https://doi.org/10.1016/j.jclepro.2021.126240, Journal of Cleaner Production, 294, 1-11 (2021)

Wright, C., y Nyberg, D., An Inconvenient Truth: How Organizations Translate Climate Change into Business as Usual, https://doi.org/10.5465/amj.2015.0718, Academy of Management Journal, 60(5), 1633-1661 (2017)

Zhai, W., Ding, J., An, X., y Wang, Z., An Optimization Model of sand and Gravel Mining Quantity Considering Healthy Ecosystem in Yangtze River, China, https://doi.org/10.1016/j.jclepro.2019.118385, Journal of Cleaner Production, 242, 1$11(2020)$ 